\title{
Line Matching Method Based on Related Points and Geometric Constraints
}

\author{
Xiang Fu, Yi Jiang, Yong Huang, Guimei Zhang*, Ying Chen \\ Institute of Computer Vision, Nanchang Hangkong University, Nanchang, 330063, China. \\ * Corresponding author. Tel.: 0791-83953406; email: fxfb163@163.com \\ Manuscript submitted March 26, 2018; accepted May 13, 2018. \\ doi: $10.17706 /$ jcp.13.11.1279-1289
}

\begin{abstract}
Combining related points and straight lines can increase the feature description ability of lines. However, mismatching problems exist for both points and lines when traditional methods are used. To improve line matching accuracy, this paper proposes a line matching method based on related points and geometric constraints. First, a statistical histogram of matched point pair distance ratios is used to eliminate mismatched SIFT feature points. After line extraction, related points are chosen and used to construct affine invariants describing line features. Lines are subsequently coarsely matched based on affine invariant similarities. Finally, an affine transformation model is calculated and lines are finely matched with line angle and distance constraints. Experimental results demonstrate the method is suitable for images with affine transformation and can obtain more matched line pairs with high accuracy than traditional methods. The proposed method considers both local and global geometric line characteristics and obtains good line matching results for images with complex or simple textures.
\end{abstract}

Key words: Line matching, line extraction, SIFT feature points, distance ratio histogram, affine invariants, geometric constraints.

\section{Introduction}

Image matching is an important task in the computer vision field, laying the foundation for applications such as target tracking, image stitching, and three-dimensional reconstruction. Generally, image matching methods can be divided into two categories: gray-scale intensity-based and feature-based. The former often uses a gray value matrix to describe image information. Though these methods can obtain subpixel precision results, they are highly sensitive to texture changes and geometric distortions. Such situations typically invalidate gray-scale intensity-based matching methods. Conversely, image feature-based matching methods can overcome these shortcomings. Image features are usually extracted from local image regions such as points and lines. The development of matching methods based on feature points is already relatively mature, including SURF, DAISY, and SIFT [1]. Point descriptors used in image matching usually consist of pixel distribution information in local regions and have certain invariances for some image transformations, such as affine, scaling and translation.

The results of the feature point-based image matching methods comparisons in [1] and [2] show the SIFT method is superior to other feature point-based methods in most situations. However, feature point-based methods performed poorly in some situations. One example of this is artificial building scenes where local feature points cannot describe global structure information well but plenty of straight lines exist. Contour lines, which can effectively represent the global structure and are robust to texture changes or shortages, 
can describe these scenes and objects. Straight line matching is thus feasible and useful in these scenes.

Most line matching methods can be divided into two classes based on the utilized features: line features only, or lines combined with related points. Line feature-based methods can match single or grouped lines. Single line matching typically uses neighboring image information such as support region [3]-[5] and color [6]; however, because these information is sensitive to image changes (such as time discrepancies in image capture) and single line lacks effective geometric properties, its performance is poor. Line group-based matching methods first divide the lines in an image into groups based on the satisfaction of selected conditions. This allows more features to be considered, increasing the ability to distinguish between lines with features such as intersection points and angles [7]-[9], which are used to match lines between different groups.

Another type of line matching method combines lines using related points [10], [11] by first applying an existing point matching approach to identify a group of matching points, then constructing an invariant based on points and lines to distinguish lines in the two images. Bin Fan et al. [12] constructed affine and projective invariants based on points and lines to match lines in transformed images. These methods, however, do not restrict features points, sometimes mismatched points may cause deviations in corresponding lines.

This paper proposes a line matching method based on related points and geometric constraints. As the information from a single line is insufficient for discriminating between lines, lines are combined using related feature points, which are extracted and matched by the robust SIFT algorithm. Mismatched feature points are eliminated based on scale invariances in SIFT. Points satisfying the given conditions are selected as line-related points. Lines are coarsely matched by measuring the similarity of invariants constructed based on lines and related points. Finally, an image transformation model is calculated and lines are finely matched based on geometric constraints.

The algorithm block diagram is shown in Fig. 1, with dashed boxes delineating the four main parts. The improved line extraction method is introduced in Section 2: lines are first extracted using the line segment detector (LSD) algorithm, with short straight-line segments undergoing further processing and fitting to maintain better object contour and scene structure integration. In Section 3, feature points are preprocessed and mismatched points are removed via new methods. Feature points are extracted and matched by the SIFT algorithm, and mismatched points are eliminated based on SIFT scale invariances. Section 4 describes the selection of line-related points satisfying selected conditions and coarse matching of lines using constructed invariants based on lines and related points. Lines are finely matched based on geometric constraints in Section 5, where coarsely matched lines are further evaluated using their degree of collinearity based on the transformation model.

\section{Line Extraction}

The LSD algorithm [13] produces results with subpixel accuracy, requires no parameter tuning, and is suitable for any digital image. LSD acquires edge line features according to the consistency of gradient directions. However, noise along edges may alter the gradient direction, leading to broken line segments. This section describes a line segment fitting method to address such situations. As shown in Fig. 2, $L_{1}$ and $L_{2}$ are two LSD-extracted lines, and $L_{3}$ is the candidate fitted line of $L_{1}$ and $L_{2}$. When $L_{1}, L_{2}$ and $L_{3}$ satisfy following conditions, $L_{1}$ and $L_{2}$ are replaced by $L_{3}$ :

$$
\left\{\begin{array}{c}
d\left(L_{1}, L_{2}\right)<10 \\
a\left(L_{1}, L_{2}\right)<5^{\circ} \\
a\left(\overline{G D_{L 1}}, \overline{G D_{L 2}}\right)<5^{\circ} \\
\overline{G M_{L 1}} \approx \overline{G M_{L 2}} \approx \overline{G M_{L 3}}
\end{array}\right.
$$


where $d\left(L_{1}, L_{2}\right)<10$ implies the distance between the end points of the two lines is small; $a\left(L_{1}, L_{2}\right)$ is the intersection angle between the two lines, which should approach collineation; $a\left(\overline{G D_{L 1}}, \overline{G D_{L 2}}\right)<5^{\circ}$ denotes the average gradient direction of the two lines, which should be similar, and $\overline{G M_{L 1}} \approx \overline{G M_{L 2}} \approx$ $\overline{G M_{L 3}}$ indicates the average gradient magnitudes of all three lines are approximately equal.

For the original image shown in Fig. 3(a), the LSD line extraction results are shown in Fig. 3(b). Fig. 3(c) presents the results following line segment fitting using the method proposed above. The red boxes in Fig. 3 show the straight-line representations of the table, sofa pillow, picture frame on the wall, and door frame contours are better integrated after short line fitting.

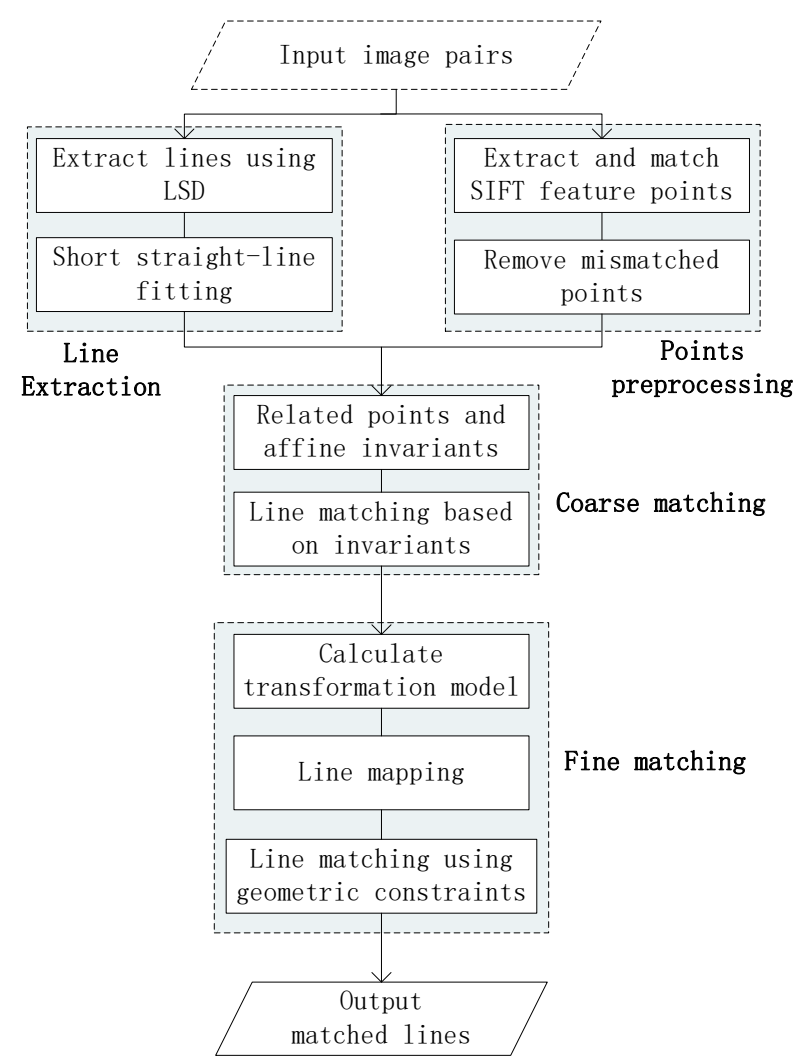

Fig. 1. Block diagram of proposed algorithm.

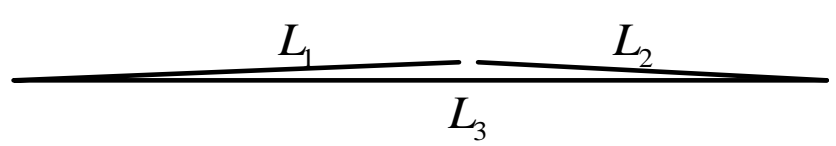

Fig. 2. Illustration of fitting line segments.

\section{Feature Point Preprocessing}

SIFT is a feature descriptor based on local neighborhood information. The mismatching probability for SIFT-based feature point matching methods increases when images contain repeating or similar textures. As point mismatches lead to matching deviations in related lines, eliminating mismatched points is critically important for subsequent line matching.

The initial SIFT-based feature matching produces matching point sets $\{R\}$ and $\{L\}$ from two images. The distance between any two points $R_{i}$ and $R_{j}$ from set $\{R\}$ is defined as:

$$
\operatorname{Dis}\left(R_{i}, R_{j}\right)=\sqrt{\sum_{m=1}^{128}\left(R_{i m}-R_{j m}\right)^{2}}
$$


where $m=1 \sim 128$ is the dimension of SIFT feature vector. For points $L_{i}$ and $L_{j}$ from set $\{L\}$, the definition is written:

$$
\operatorname{Dis}\left(L_{i}, L_{j}\right)=\sqrt{\sum_{m=1}^{128}\left(L_{i m}-L_{j m}\right)^{2}}
$$

Distance ratio is defined as:

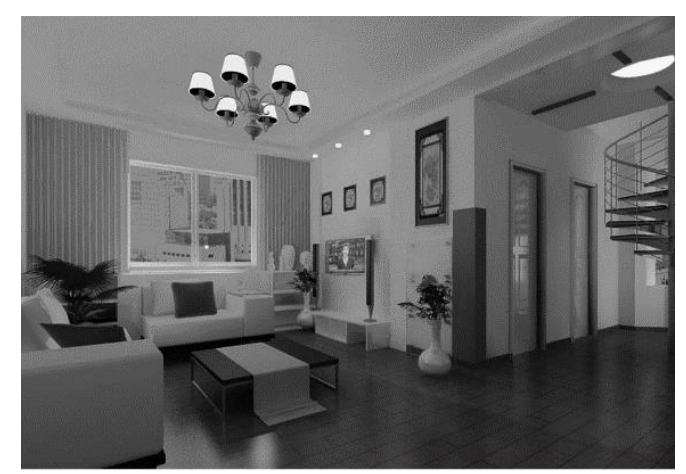

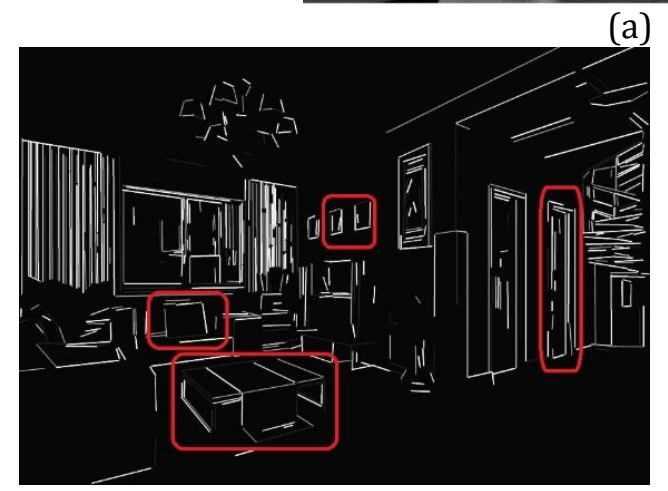

(b)

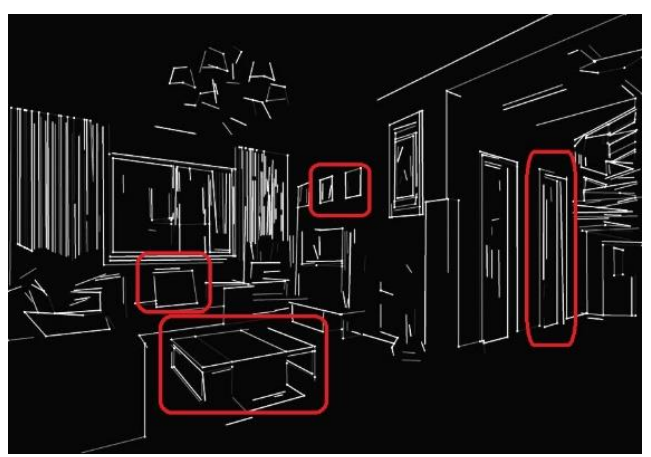

(c)

Fig. 3. Line extraction results: (a) original image, (b) lines extracted by LSD, and (c) results after line segment fitting.

$$
D_{i j}=\frac{\operatorname{Dis}\left(R_{i}, R_{j}\right)}{\operatorname{Dis}\left(L_{i}, L_{j}\right)}
$$

where $R_{k}$ and $L_{k}(k=i, j)$ are a pair of matched feature points from the two images.

The ratio defined in (4) is of considerable significance in the proposed method. Based on SIFT invariances, correctly matched point pairs should have an approximately equal ratio (equivalent to the ratio of scale transformation between the two images). The statistic histogram of distance ratios should thus be concentrated around a peak value reflecting the transformation scale between the two images. Discrete values far from this peak value correspond to mismatched points.

The results of mismatched point pair removal for images taken from different view angles are shown in Fig. 4. Several mismatched point pairs are shown in the initial matching results in Fig. 4(a). The corresponding distance ratio histogram is shown in Fig. 4(c), with a clearly defined peak value and distant discrete values. Fig. 4(d) is the histogram after discrete values are removed, and the corresponding matching effect is shown in Fig. 4(b). Most mismatched point pairs in Fig. 4(a) were eliminated successfully. A similar process and results, obtained for images captured at different times, are shown in Fig. 5.

\section{Coarse Matching}

\subsection{Related Points and Affine Invariants}

Suppose $H$ is affine transformation matrix between two images, $\left(l, l^{\prime}\right)$ is a pair of lines, $\left(X_{1}, X_{1}^{\prime}\right)$ and 
$\left(X_{2}, X_{2}^{\prime}\right)$ are two pairs of points from the two images, and the related homogeneous coordinates are represented as $\left(\widetilde{X_{1}}, \widetilde{X_{1}^{\prime}}\right)$ and $\left(\widetilde{X_{2}}, \widetilde{X_{2}^{\prime}}\right)$. The following transformation formulas apply:

$$
\begin{gathered}
l^{\prime}=s H^{-T} l \\
\widetilde{X_{l}^{\prime}}=H \widetilde{X}_{l}, i=1,2
\end{gathered}
$$

where $s$ is the scale factor between the two images. Within each image, the distance ratio can be defined as

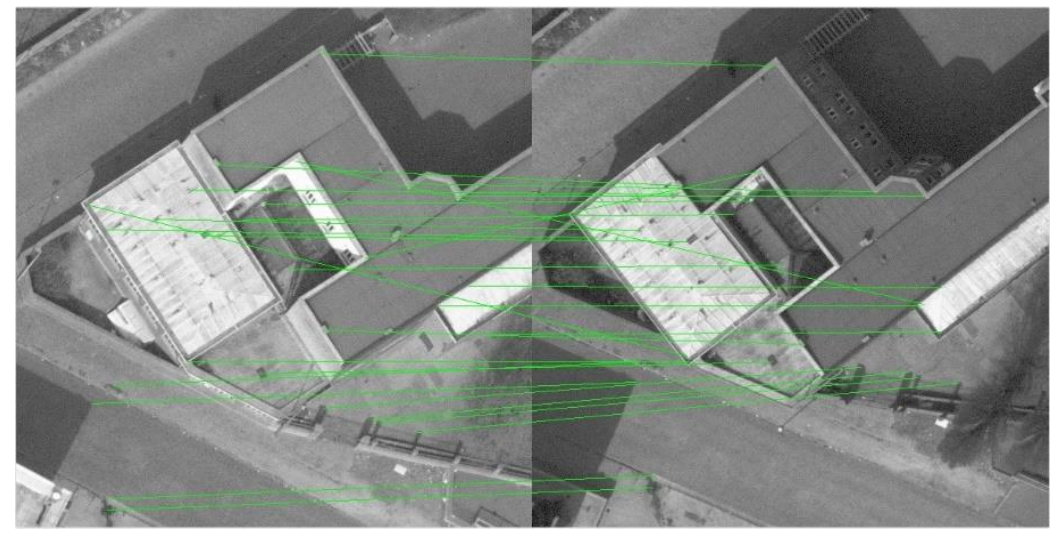

(a)

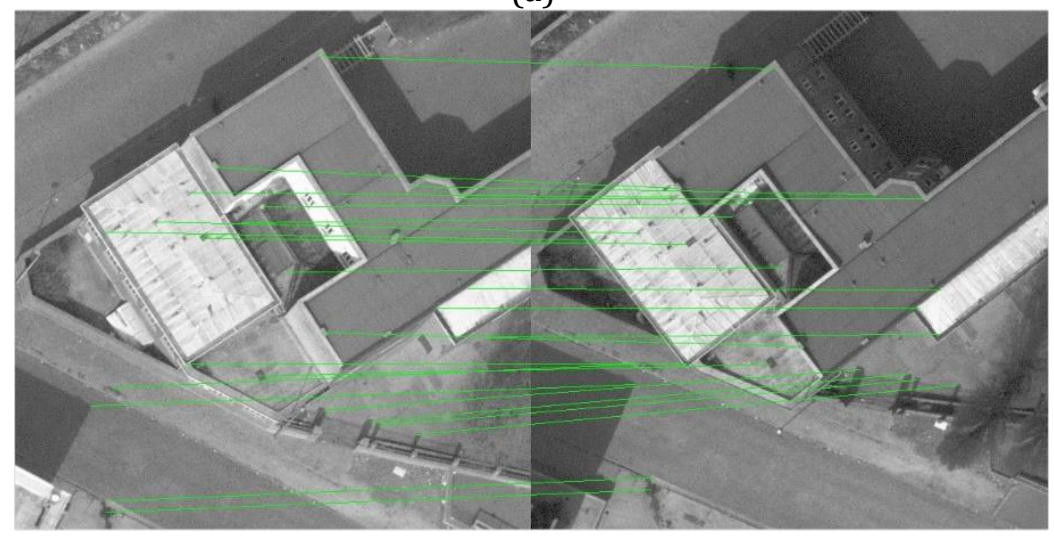

(b)

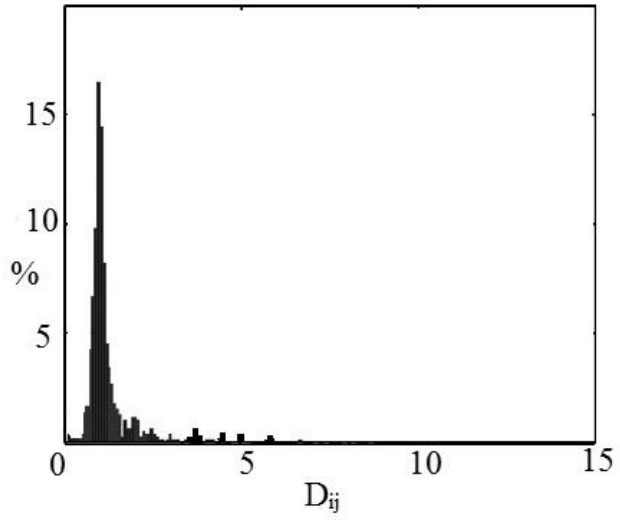

(c)

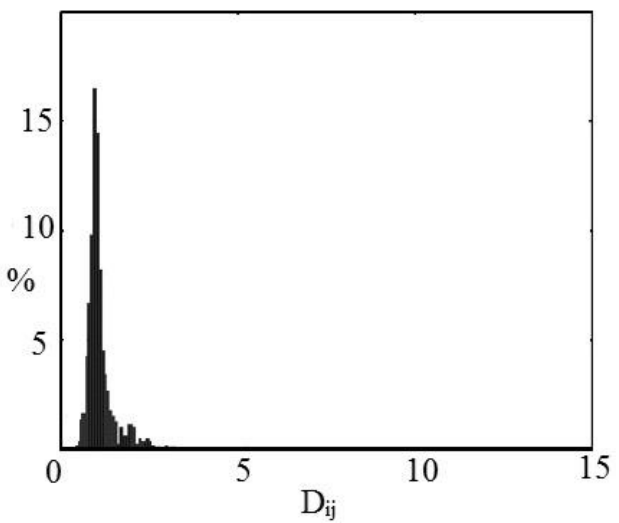

(d)

Fig. 4. Removing mismatched point pairs based on distance ratio histogram for images with different view angles: (a) initial SIFT matching results, (b) results after removing mismatched point pairs, (c) distance ratio histogram of (a), and (d) distance ratio histogram of (b). 


$$
\begin{aligned}
& D\left(X_{1}, X_{2}, l\right)=\frac{l^{T} \widetilde{X_{1}}}{l^{T} \widetilde{X_{2}}} \\
& D\left(X_{1}^{\prime}, X_{2}^{\prime}, l^{\prime}\right)=\frac{l^{\prime T} \widetilde{X_{1}^{\prime}}}{l^{\prime T} \widetilde{X_{2}^{\prime}}}
\end{aligned}
$$

where $l^{T} \widetilde{X_{\mathrm{k}}},(k=1$ and 2$)$ represents the distance from point $X_{k}$ to line $l$. Substituting (5) and (6) into (7) and (8) produces

$$
D\left(X_{1}, X_{2}, l\right)=D\left(X_{1}^{\prime}, X_{2}^{\prime}, l^{\prime}\right)
$$

This indicates the distance ratio between different points and a line is an affine invariant.

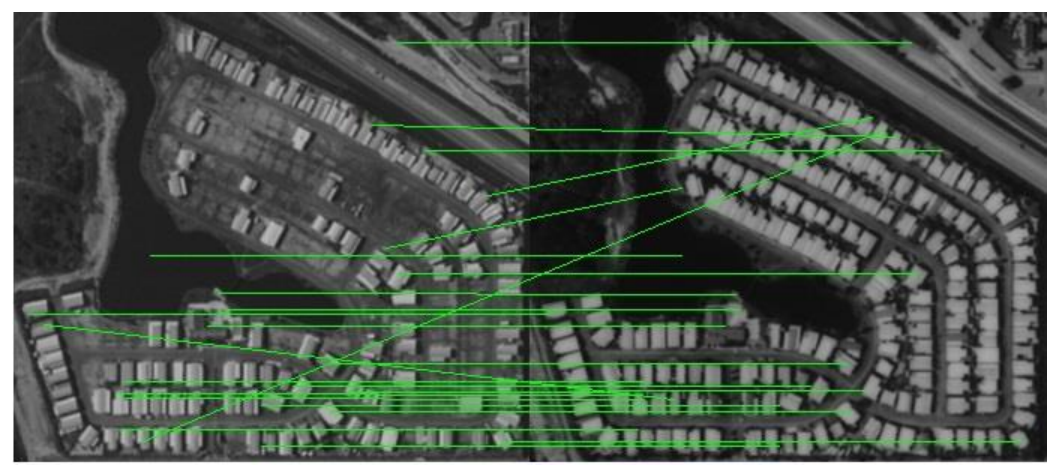

(a)

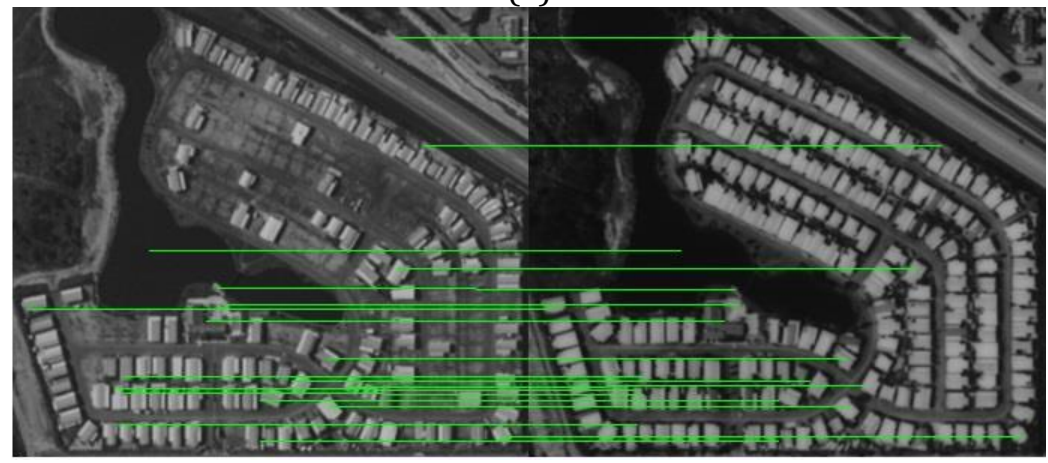

(b)

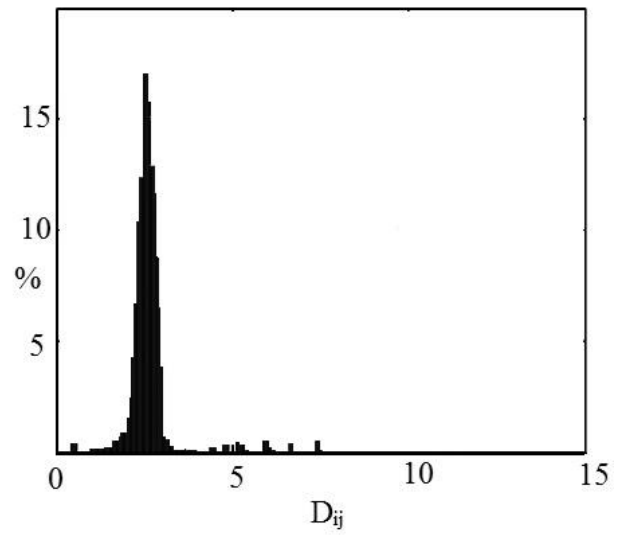

(c)

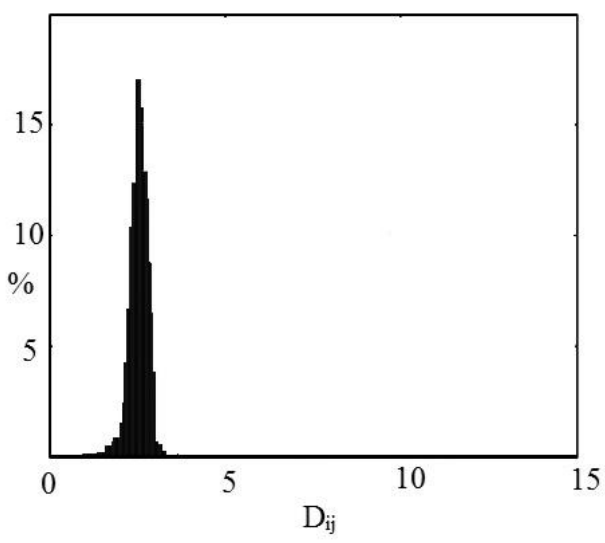

(d)

Fig. 5. Removing mismatched point pairs based on distance ratio histogram for images captured at different times: (a) initial SIFT matching results, (b) results after removing mismatched point pairs, (c) distance ratio histogram of (a), and (d) distance ratio histogram of (b).

The two nearest feature points of a straight line are defined as its related points; however, related points cannot be located on the line. The related points of a single line, extracted in Section 2, are selected from the 
feature points reprocessed in Section 3. An affine invariant constructed from a line and its related points can be used to describe the features of the line. The similarity measurement of line features is defined as

$$
\operatorname{sim}\left(l, l^{\prime}\right)=e^{-\left\|D\left(X_{1}, X_{2}, l\right)-D\left(X_{1}^{\prime}, X_{2}^{\prime}, l^{\prime}\right)\right\|}
$$

where $X_{1}$ and $X_{2}$ are the related points of line $l$ in one image, $X_{1}^{\prime}$ and $X_{2}^{\prime}$ are the related points of line $l^{\prime} \quad$ in another image, and $X_{i}$ and $X_{i}^{\prime} \quad(i=1$ and 2$)$ are a pair of matching points obtained in section 3. If lines $l$ and $l^{\prime}$ match, the value of $\left\|D\left(X_{1}, X_{2}, l\right)-D\left(X_{1}^{\prime}, X_{2}^{\prime}, l^{\prime}\right)\right\|$ should approach 0 and $\operatorname{sim}\left(l, l^{\prime}\right)$ should be large. Small $\operatorname{sim}\left(l, l^{\prime}\right)$ values indicate that line $l$ does not match $l^{\prime}$.

\subsection{Coarse Matching Results}

A coarse determination of matching between lines $l$ and $l^{\prime}$ can be performed based on a threshold $\operatorname{sim}\left(l, l^{\prime}\right)$ value. This is called coarse matching as larger thresholds ensure correct matching but may ignore some matching line pairs, but smaller thresholds lead to numerous mismatched results. The threshold here was set to $\operatorname{sim}=0$.9. Corresponding results are shown in Fig. 6. Fig. 6(a) includes images captured from two different view angles, with most lines correctly matched. The situation of "one-to-many" occurred for some lines, such as No. 13, 18 and 26, marked with red boxes. Fig. 6(b) shows the matching results of the two aviation images with affine transformation. However, two among the 27 line pairs remained matched with more than one line. This issue is further processed in the fine matching stage.
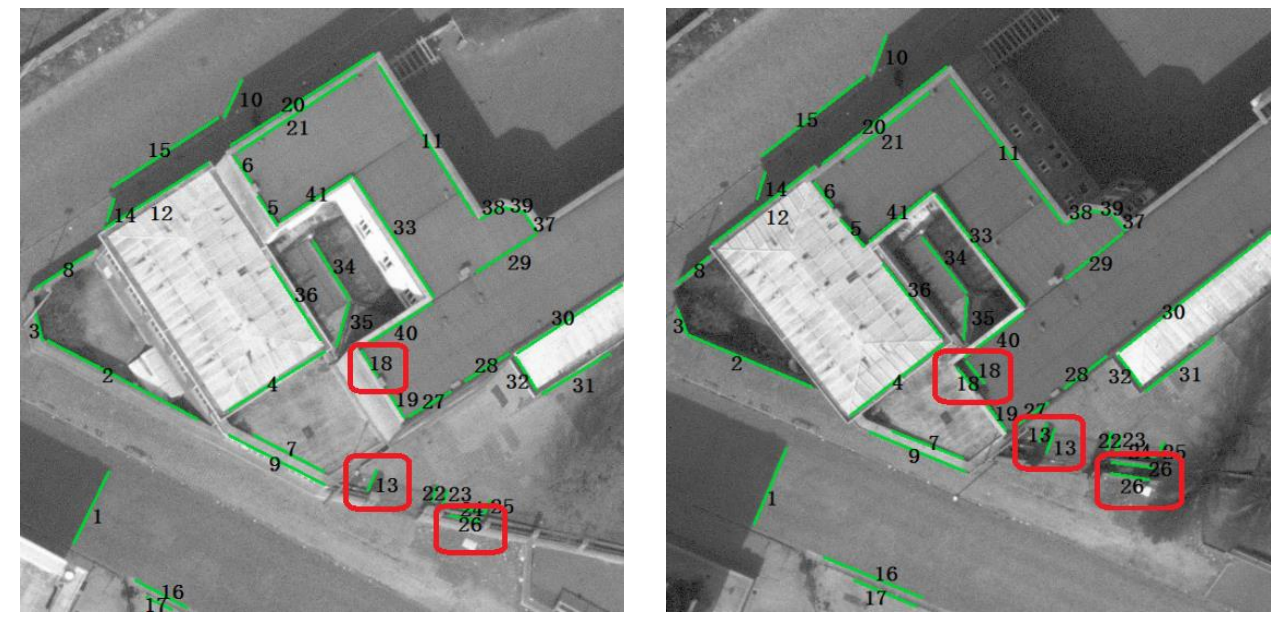

(a)
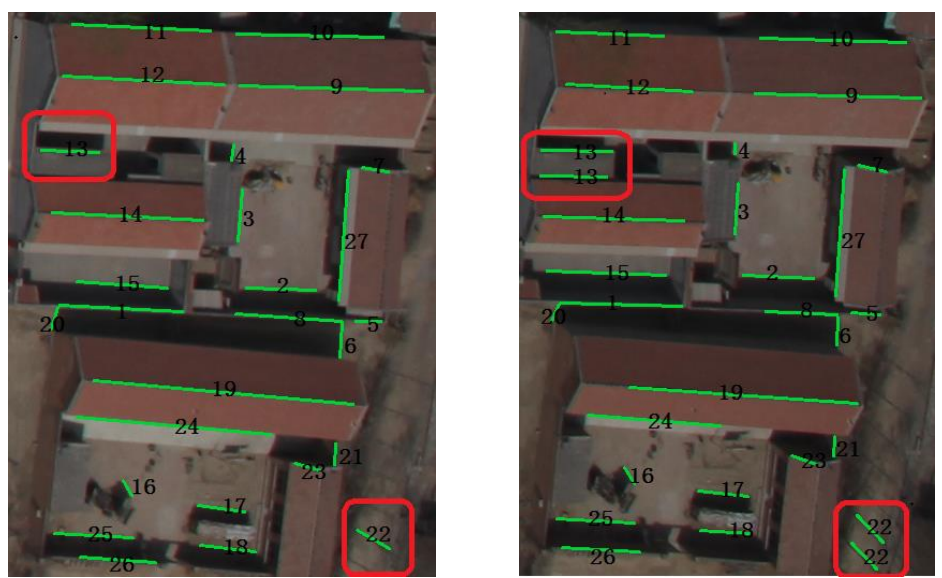

(b)

Fig. 6. Coarse matching results: (a) aviation images from different angles, (b) aviation images with affine transformation. 


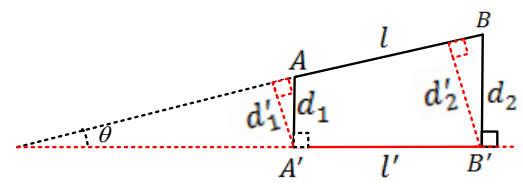

Fig. 7. Illustration of line geometry constraints.

\section{Fine Matching}

\subsection{Line Matching Based on Geometric Constraints}

To solve the "one-to-many" problem that arose during coarse matching, geometric constraints are used to improve matching accuracy in this section.

First, transformation model parameters were calculated based on matching feature points from section 3 . In theory, these parameters can be calculated based on any three pairs of points. Though mismatched points were removed using the distance ratio histogram, the possibility of mismatched points persists. To further avoid mismatched point-related errors, the transformation model parameters were calculated ten times using randomly selected different point sets. The mean value of parameters was then calculated, and the five parameter sets differing the most from the mean value are discarded. A new mean value is calculated from the preserved five parameter sets, and this is used as the final transformation model parameter.

Lines are then mapped between images based on the transformation model parameters. As shown in Fig. 7, $\left(l, l^{\prime}\right)$ is a matched line pair obtained as described in section 4, where $A$ and $B$ are the two end points of line $l, A^{\prime}$ and $B^{\prime}$ are end points of $l^{\prime}, \theta$ represents the intersection angle between $l$ and $l^{\prime}$, and the distance between $l$ and $l^{\prime}$ is defined as

$$
D=\sqrt{d_{1}^{2}+d_{2}^{2}+d_{1}^{\prime 2}+d_{2}^{\prime 2}}
$$

where $d_{1}^{\prime}$ and $d_{2}^{\prime}$ are the distances from $A^{\prime}$ and $B^{\prime}$ to line $l$, respectively, and $d_{1}$ and $d_{2}$ are the distances from $A$ and $B$ to $l^{\prime}$, respectively. If $l$ and $l^{\prime}$ match each other, $\theta$ and $D$ should be very small; for example, $\theta<5^{\circ}$ and $D<10$. A small $D$ can guarantee small values of $d_{1}, d_{2}, d_{1}^{\prime}$ and $d_{2}^{\prime}$. When both $\theta$ and $D$ are small, $l$ and $l^{\prime}$ are approximately collinear. If more than one line in the second images satisfies this condition for a line in the first image, the line with the smallest $D$ is chosen as the final matching result.

\subsection{Fine Matching Results}

The coarsely matched results in Fig. 6 were further processed by the fine matching method, with the corresponding results shown in Fig. 8 demonstrating that the "one-to-many" problem was solved successfully. Fig. 9 shows the matching results for low-texture images of an indoor scene, where the proposed coarse-to-fine method also performed well.

The objective matching results are given in Table 1, where the proposed method is compared with other two methods from literature: the mean-standard deviation line descriptor (MSLD) [3] and line point invariants (L-P) [12]. The MSLD matches lines based on their support region and L-P uses invariants based on lines and feature points. Three image pairs used for comparison are all with some degree of scale transformation. The performance of MSLD is relatively poor, such that the total matched number of line pairs is less and the mismatched ones are more than other two methods because the support region is sensitive to image transformation and texture changes. L-P obtained more matched pairs and fewer mismatched results; however, mismatching remained present because mismatched feature points were not removed at the early stage. The proposed method avoided the mismatching problem by eliminating mismatched points based on the distance ratio histogram and using fine matching processing based on 
geometric constraints.

Table 1. Line Matching Results

\begin{tabular}{|c|c|c|c|c|}
\hline \multirow{2}{*}{ Image pairs } & \multirow{2}{*}{$\begin{array}{l}\text { Number of lines extracted from } \\
\text { two images }\end{array}$} & \multicolumn{3}{|c|}{ Total number of matched pairs (Number of mismatched pairs) } \\
\hline & & MSLD [3] & L-P [12] & Proposed \\
\hline Fig. 8(a) & $65 / 78$ & $39(7)$ & $48(5)$ & $41(0)$ \\
\hline Fig. 8(b) & $42 / 51$ & $25(6)$ & $31(3)$ & $27(0)$ \\
\hline Fig. 9 & $31 / 39$ & $8(2)$ & $18(2)$ & $20(0)$ \\
\hline
\end{tabular}
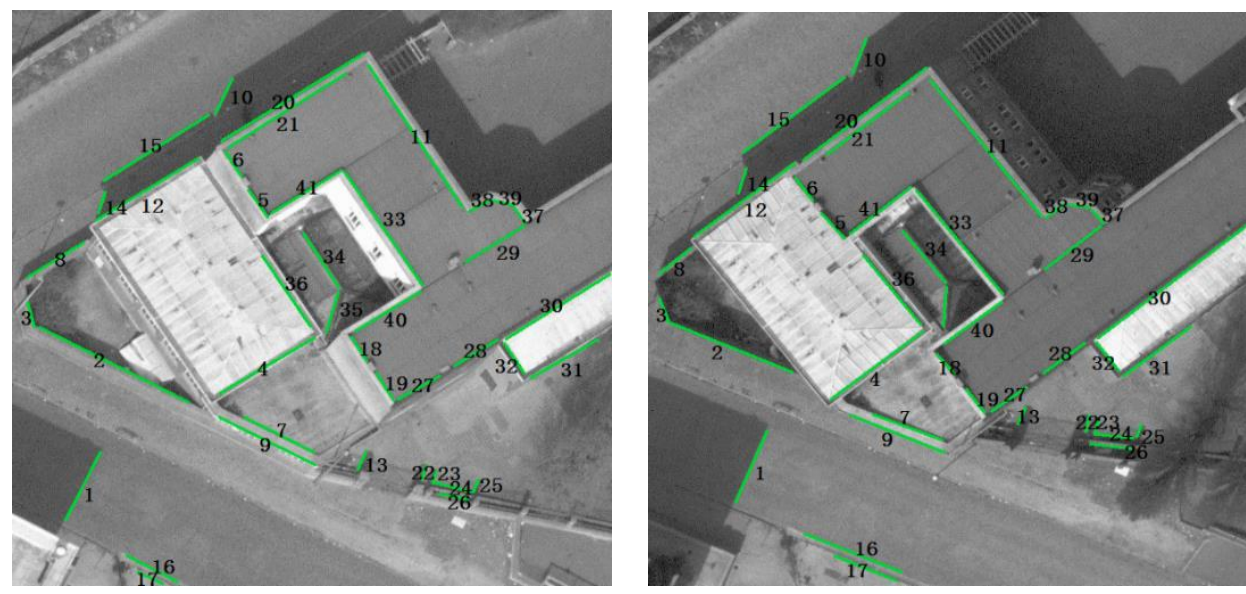

(a)
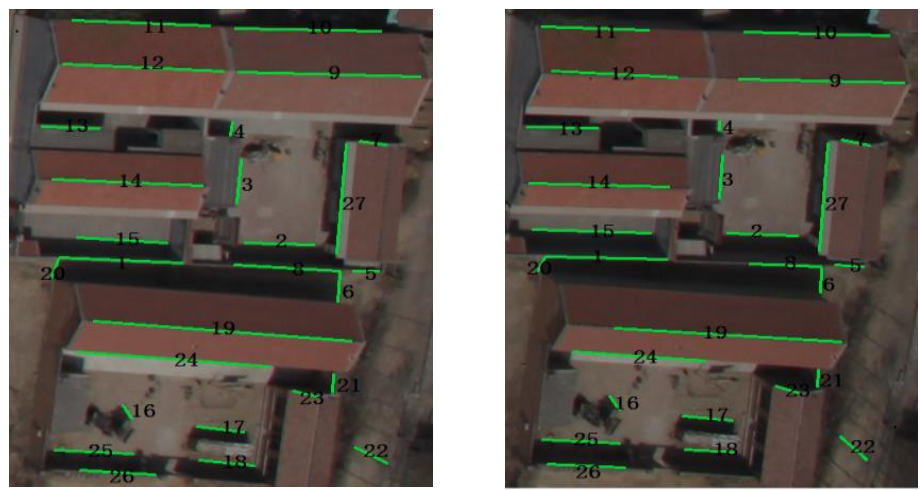

(b)

Fig. 8. Fine matching results: (a) Aviation images from different view angles, (b) aviation images with affine transformation.
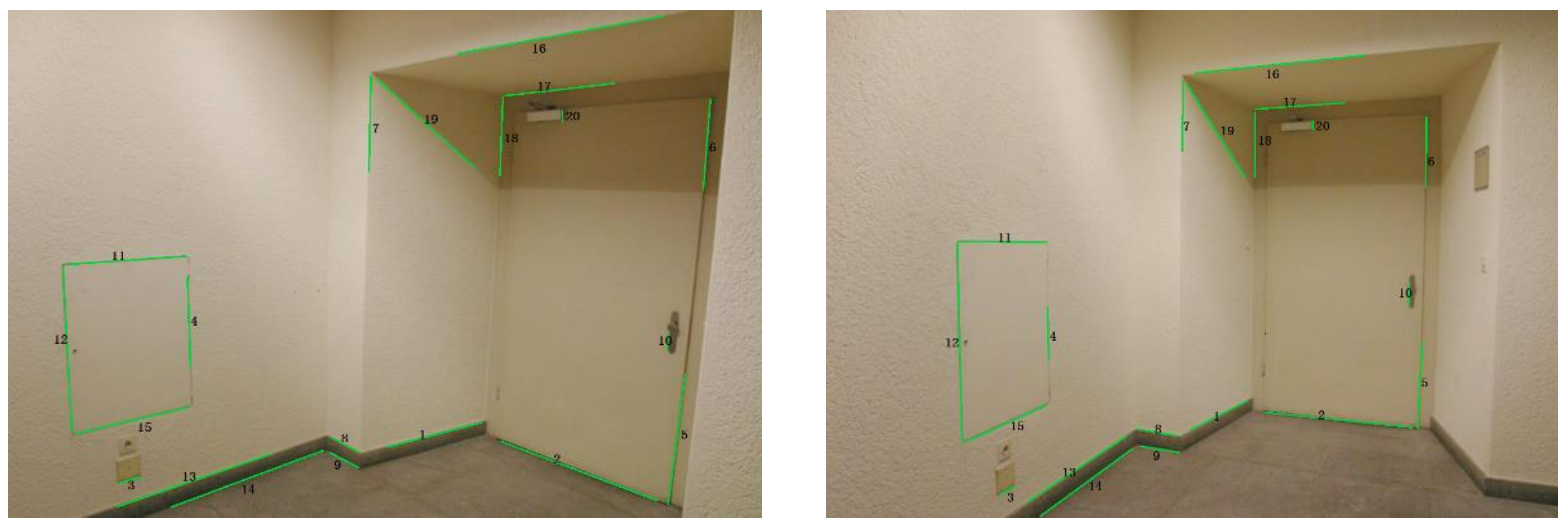

Fig. 9. Matching results for low-texture images of indoor scenes. 


\section{Conclusion}

This paper proposed a line matching method based on related points and geometric constraints. Though the information from a single line is insufficient for discriminating between lines, this can be done by combining a line with related points within its neighboring locations. Statistical histograms of distance ratios between matched feature points are effective for reducing mismatched points, and mismatched lines can be eliminated by constructing geometric constraints based on the affine transformation model. This method is suitable for artificial building scenarios with numerous straight lines. It is robust to the affine transformation of images and can also obtain good matching results for low-texture images.

\section{Acknowledgment}

This work was supported in part by the National Natural Science Foundation of China (61662049, 61762067, 61763033), China Scholarship Council (201608360163) and the Natural Science Foundation of Jiangxi Province (20161BAB212034).

\section{References}

[1] Rani, R., Kumar, R., \& Singh, A. P. (2018). An empirical evaluation of translational and rotational invariance of descriptors and the classification of flower dataset. Pattern Analysis and Applications, 21(1), 1-18.

[2] Khan, N., McCane, B., \& Mills, S. (2015). Better than SIFT? Machine Vision and Applications, 26(6), 819-836.

[3] Wang, Z., Wu, F., \& Hu, Z. (2009). MSLD: A robust descriptor for line matching. Pattern Recognition, 42(5), 941-953.

[4] Zhang, L., \& Koch, R. (2013). An efficient and robust line segment matching approach based on LBD descriptor and pairwise geometric consistency. J. Vis. Commun. Image Represent, 24(7), 794-805.

[5] Zhang, Y., \& Qu, H. S. (2014). Rotation invariant feature lines transform for image matching. Journal of Electronic Imaging, 23(5), 053002.

[6] Bay, H., Ferrari, V., \& Gool, L. V. (2005). Wide-baseline stereo matching with line segments. Proceedings of the 2005 IEEE Computer Society Conference on Computer Vision and Pattern Recognition (pp. 329-336).

[7] Li, K., Yao, J., \& Lu, X., et al. (2016). Hierarchical line matching based on line-junction-line structure descriptor and local homography estimation. Neurocomputing, 184, 207-220.

[8] Ramalingam, S., Antunes, M., \& Snow, D., et al. (2015). Line-sweep: Cross-ratio for wide-baseline matching and 3D reconstruction. Proceedings of the 2015 IEEE Conference on Computer Vision and Pattern Recognition (pp. 1238-1246).

[9] Zhang, J., Zhao, Q., \& Fan, X., et al. (2012). Line matching based on characteristic ratio invariants of collinear points. Proceedings of the 2012 IEEE Conference on Digital Home (pp. 268-273).

[10] Zeng, J., Zhan, L., \& Fu, X., et al. (2016). Straight line matching method based on line pairs and feature points. IET Computer Vision, 10(5), 459-468.

[11] Chen, M., Shao, Z., \& Liu, C., et al. (2013). Scale and rotation robust line-based matching for high resolution images. Optik-International Journal for Light and Electron Optics, 124(22), 5318-5322.

[12] Fan, B., Wu, F., \& Hu, Z. (2012). Robust line matching through line-point invariants. Pattern Recognition, 45(2), 794-805.

[13] Von, G. R., Jakubowicz, J., \& Morel, J. (2010). LSD: A fast line segment detector with a false detection control. IEEE Transactions on Pattern Analysis and Machine Intelligence, 32(4), 722-732. 


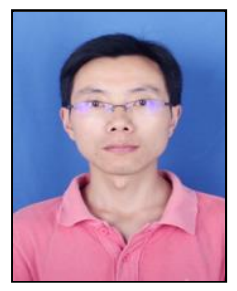

Xiang Fu received his PH.D degree from Xidian University in 2008. Now he is an associate professor at Nanchang Hangkong University, his interesting research area is computer vision, image processing and pattern recognition.

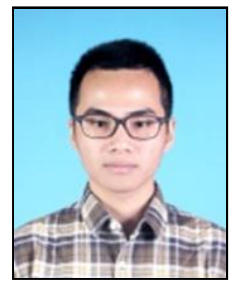

Yi Jiang received his bachelor degree in Nanchang Hangkong University. $\mathrm{He}$ is a postgraduate at Nanchang Hangkong University. His main research area is computer vision and image processing.

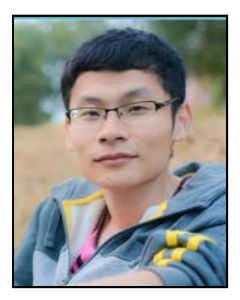

Yong Huang received his M.E. degree in Nanchang Hangkong University. His main research area is computer vision and image processing.

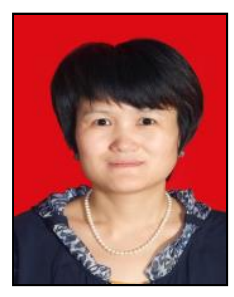

Guimei Zhang received her PH.D degree from Northwestern Polytechnical University in 2006. Now she is a professor at Nanchang Hangkong University, her interesting research area is computer vision, image processing and pattern recognition.

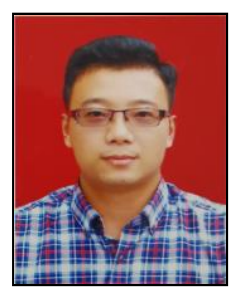

Ying Chen received his Ph.D degree from Jilin University, Changchun, China in 2014. He is an associate professor at Nanchang Hangkong University, Nanchang, Jiangxi, China. His main research area is biometric information recognition, information security, wireless sensor network and Internet of Things. 